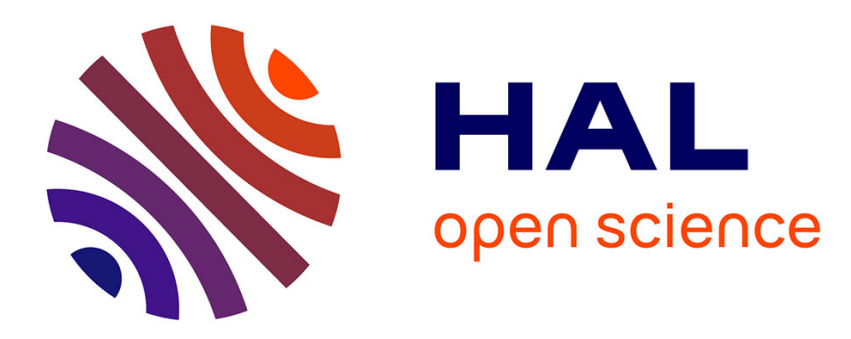

\title{
Une étude comparative de dispositifs photovoltaïques multiples
}

\author{
J.R. Leguerre, M. Leroy
}

\section{To cite this version:}

J.R. Leguerre, M. Leroy. Une étude comparative de dispositifs photovoltaïques multiples. Revue de Physique Appliquée, 1984, 19 (10), pp.889-903. 10.1051/rphysap:019840019010088900 . jpa00245281

\section{HAL Id: jpa-00245281 https://hal.science/jpa-00245281}

Submitted on 1 Jan 1984

HAL is a multi-disciplinary open access archive for the deposit and dissemination of scientific research documents, whether they are published or not. The documents may come from teaching and research institutions in France or abroad, or from public or private research centers.
L'archive ouverte pluridisciplinaire HAL, est destinée au dépôt et à la diffusion de documents scientifiques de niveau recherche, publiés ou non, émanant des établissements d'enseignement et de recherche français ou étrangers, des laboratoires publics ou privés. 
Classification

Physics Abstracts

73. $40 \mathrm{~L}$

\title{
Une étude comparative de dispositifs photovoltaïques multiples
}

\author{
J. R. Leguerre et M. Leroy
}

Laboratoire d'Automatique et d'Analyse des Systèmes du Centre National de la Recherche Scientifique, 7, avenue du Colonel Roche, 31077 Toulouse Cedex, France

(Reçu le 24 mai 1983, révisé le 23 mai 1984, accepté le 16 juillet 1984)

Résumé. - Un calcul précis des valeurs des largeurs de bande interdite $E g_{k}$ de matériaux hypothétiques et du rendement maximum $\eta_{\max }$ correspondant a été effectué pour des associations multicolores de photopiles couplées en mode dichroïque, tandem ou cascade. L'effet d'un couplage optique par un miroir unique entre plusieurs cellules solaires isolées et/ou associées en mode tandem ou cascade a été particulièrement analysé ainsi que l'influence d'un couplage électrique par un simple fil conducteur entre les photopiles.

Une simulation a été réalisée avec des matériaux réels tels que $\mathrm{Ge}, \mathrm{Si}, \mathrm{AsGa}$. Un résultat caractéristique de cette étude est que le couplage par un miroir dichroïque d'une cellule et d'une structure cascade est particulièrement intéressant sur le plan du rendement et les valeurs des $E g_{k}$ sont pratiquement insensibles au niveau de concentration solaire.

Abstract. - A precise calculation of the values of gaps $E g_{k}$ of hypothetical materials and of the corresponding maximum efficiency $\eta_{\max }$ was carried out for multigap solar cells systems coupled in splitting, tandem or cascade mode. The effects of an optical coupling by a dichroic mirror between independent solar cells or/and cells coupled in tandem or cascade mode has been analysed in particular in addition to the influence of an electrical coupling of a simple conducting wire between the cells.

A simulation program has been performed on materials as $\mathrm{Ge}, \mathrm{Si}, \mathrm{GaAs}$. One particular result of our study is that the coupling by a dichroic mirror between an independent cell and a cascade structure gives an interesting efficiency and the values of gaps $E g_{k}$ are practically insensitive to the level of solar concentration.

\section{Nomenclature}

$C R \quad$ : Facteur de concentration solaire

$D_{\mathrm{n}} \quad$ : Constante de diffusion des électrons

$D_{\mathrm{p}} \quad$ : Constante de diffusion des trous

$E g_{k} \quad$ : Largeur de bande interdite ou « gap » de la cellule $k$

$J \quad:$ Densité du courant qui traverse la cellule

$J_{0} \quad$ : Densité du courant de saturation

$J_{L} \quad:$ Densité du courant de lumière

$k \quad$ : Constante de Boltzmann

$N_{\mathrm{A}} \quad$ : Dopage côté $\mathrm{P}$ de la jonction

$N_{\mathrm{D}} \quad$ : Dopage côté $\mathrm{N}$ de la jonction

$N_{\mathrm{ph}}(h v)$ : Nombre de photons incidents d'énergie $h v$ par unité de surface et de temps

$N_{\mathrm{c}} \quad$ : Densité effective d'états dans la bande de conduction

$N_{\mathrm{v}} \quad$ : Densité effective d'états dans la bande de valence

$q \quad$ : Charge de l'électron

$r_{k} \quad$ : Réflectance superficielle de la cellule $k$

$T \quad:$ Température absolue

$T_{\mathrm{r} k} \quad$ : Transmittance du miroir $k$
$V \quad:$ Tension aux bornes de la cellule

$\eta_{\mathrm{m}} \quad:$ Rendement maximum d'une combinaison de $k$ cellules $(k>1)$, le « gap » $E g_{1}$ n'étant pas à sa position optimale

$\eta_{\max } \quad$ : Valeur de $\eta_{\mathrm{m}}, E g_{1}$ étant à sa position optimale

$\rho_{k} \quad:$ Taux d'ombrage de la cellule $k$

$\tau_{k} \quad$ : Coefficient de transmission de la cellule $k$

$\tau_{\mathbf{n}} \quad:$ Durée de vie des électrons

$\tau_{\mathbf{p}} \quad$ : Durée de vie des trous.

\section{Introduction.}

Parmi les pertes responsables du faible rendement des photopiles solaires, celles dues à l'excès d'énergie et à la non-absorption des photons sont les plus importantes. Le concept de l'utilisation simultanée de plusieurs photopiles de matériaux différents permet dans une certaine mesure, de limiter ces pertes et d'obtenir un meilleur rendement. Les cellules peuvent être couplées entre elles en les disposant les unes derrière les autres sur le trajet optique comme dans 
le système tandem [1] ou par l'intermédiaire de miroirs dichroïques qui envoient sur chacune d'elles, une partie bien définie du spectre solaire comme dans le système dichroïque [2]. Une autre disposition est la structure cascade [3] où plusieurs jonctions $p-n$ de matériaux différents sont empilées les unes derrière les autres en formant un bloc monolithique. Les différentes cellules sont donc placées sur le faisceau optique comme dans le système tandem, mais sont aussi, par définition même du dispositif, couplées électriquement.

Les travaux théoriques antérieurs ont surtout mis en évidence les augmentations possibles de rendement de systèmes de cellules indépendantes couplées en mode tandem ou en mode dichroïque. Cependant, il faut noter l'intérêt des groupements mixtes faisant intervenir des combinaisons des trois modes de couplage : tandem, dichroïque et cascade.

Dans ce travail, nous proposons une analyse de ce problème en considérant que le couplage optique entre les cellules est effectué non seulement par l'un ou l'autre des trois modes de couplage tandem ou dichroïque ou cascade, mais encore par une combinaison des modes dichroïque et cascade ou dichroïque et tandem.

De plus, nous avons souvent considéré le cas où les cellules sont mises en série électrique par un simple fil conducteur qui relie entre elles les photopiles initialement indépendantes. Le but de cette étude est de mettre en évidence d'une part les différences qui existent entre les modes cascade et tandem couplés électriquement, d'autre part de connaître les modifications par rapport au système à cellules indépendantes lorsque sur le plan pratique on décide d'effectuer une liaison électrique série entre les éléments par un. simple fil conducteur.

Nous avons étudié des systèmes de un, deux, trois et quatre cellules constitués de matériaux hypothétiques de largeurs de bande interdite respectives $E g_{1}$, $E g_{2}, E g_{3}, E g_{4}$ avec toujours $E g_{1}>E g_{2}>E g_{3}>E g_{4}$; nous donnerons les valeurs optimales des $E g_{k}$ et les maxima de rendement de nos systèmes.

La figure 1 montre les différentes structures que nous avons étudiées; on distingue deux grandes subdivisions :

- les systèmes simples,

- les systèmes mixtes à un miroir.

Pour les premiers, nous avons considéré des cellules indépendantes en mode tandem et dichroïque et sont représentés sur les dessins numérotés (1a) et (1b) respectivement. Les dessins (1c) et (1d) représentent le cas lorsqu'elles sont reliées électriquement et le dessin (1e) celui où les cellules sont associées en mode cascade.

Pour les seconds, nous avons volontairement limité notre étude à des systèmes de cellules solaires utilisant un miroir unique; en effet, le mode dichroïque faisant intervenir deux ou trois miroirs ne semble pas trop réaliste à notre avis, le coût d'un tel ensemble pouvant devenir prohibitif. Nous avons ainsi commencé à analyser des associations à trois cellules; il faut alors noter qu'il peut exister deux types de configurations.

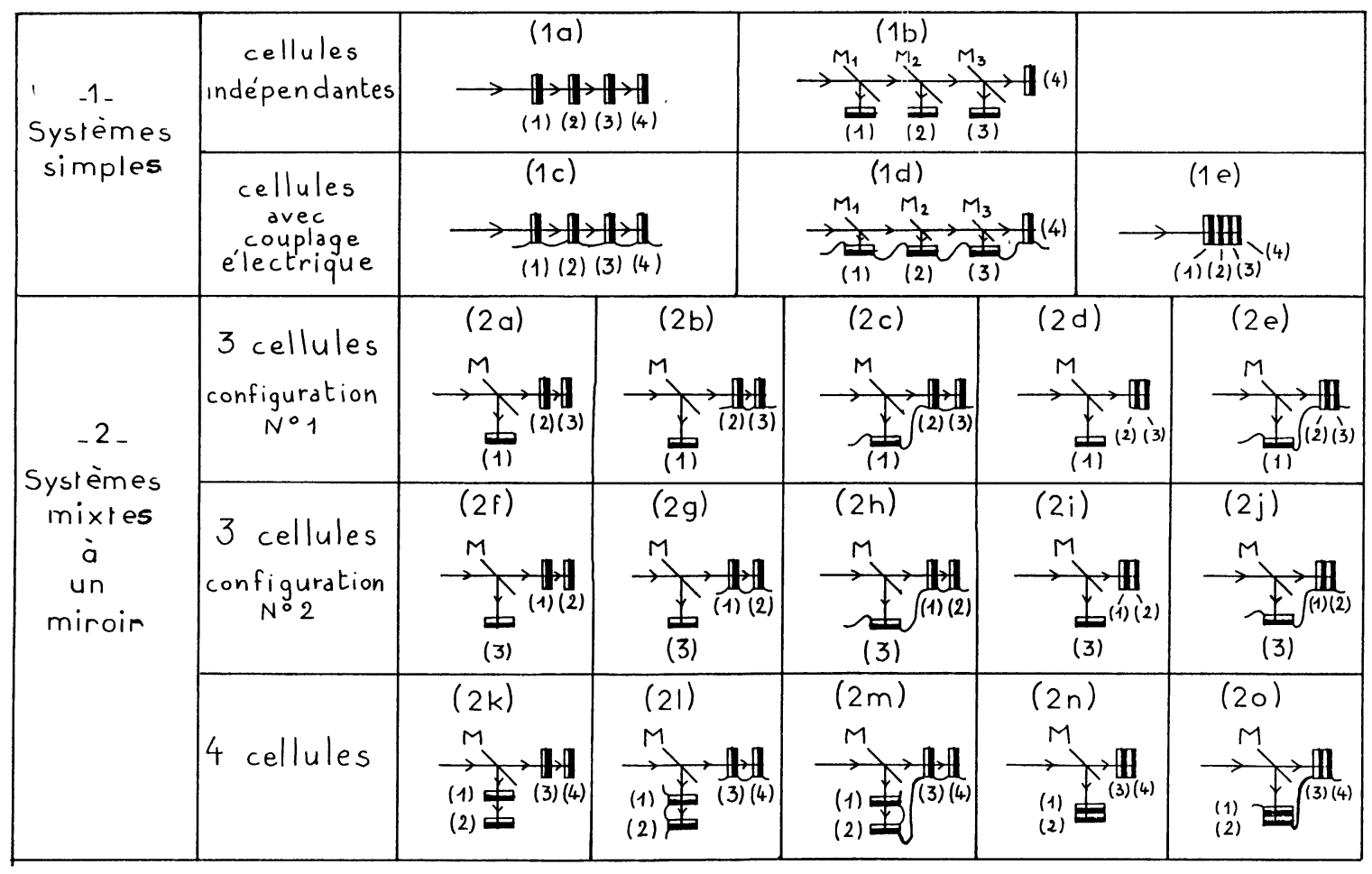

Fig. 1. - Schéma des différents groupements de cellules.

[Scheme of different systems]. 
Dans la configuration $\mathrm{n}^{0} 1$, le dessin (2a) de la figure 1 montre que la cellule 1 reçoit la partie du rayonnement lumineux d'énergie $h v \geqslant E g_{1}$ où $h v$ est le quantum d'énergie du photon. Les cellules 2 et 3 de bandes interdites respectives $E g_{2}$ et $E g_{3}$ sont en mode tandem et reçoivent la partie du rayonnement d'énergie $h v<E g_{1}$. La courbe de la transmittance $T_{\mathrm{r}}$ du miroir en fonction de l'inverse de l'énergie $h v$ du photon, doit alors avoir l'allure représentée sur la figure 2a. Dans ce travail, nous avons supposé que le miroir est idéal et ne sert qu'à effectuer une séparation spectrale. Le rendement du système ne dépendra alors que de la technologie de la configuration et du mode de liaison électrique des cellules. Le dessin (2b) de la figure 1 considère le cas précédent où les cellules 2 et 3 sont maintenant reliées par un fil électrique tandis que $(2 \mathrm{c})$ représente le cas où les trois cellules sont liées par un fil électrique. Le dessin (2d) reprend le groupement de (2a) dans lequel le tandem de cellules 2-3 est remplacé par une cascade; le dessin (2e) considère alors l'effet d'une liaison électrique entre la cascade 2-3 et la cellule 1 . La configuration $\mathrm{n}^{\circ} 2$

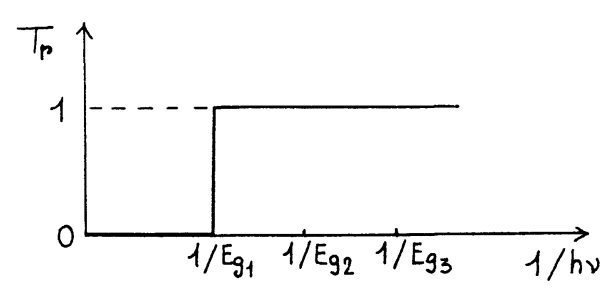

(b)

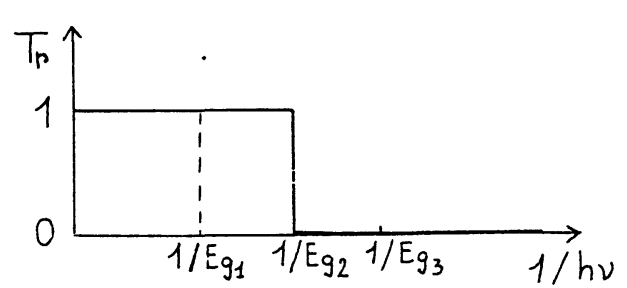

(c)

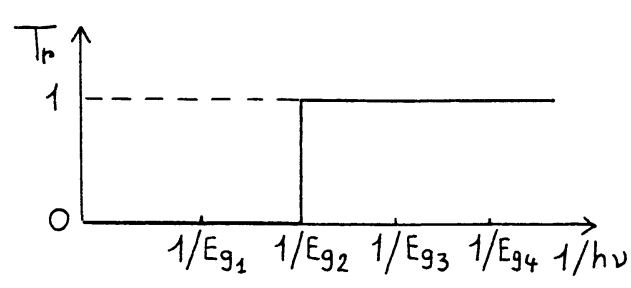

Fig. 2. - Transmittance $T_{\mathrm{r}}$ du miroir dichroïque en fonction de l'inverse de l'énergie $h v$ du photon pour : a) systèmes mixtes de 3 cellules, configuration 1 ; b) systèmes mixtes de 3 cellules, configuration $2 ; c$ ) systèmes de 4 cellules.

[Transmittance $T_{\mathrm{r}}$ of the dichroic mirror as a function of the inverse of photon energy $h v$ for : a) combined systems of 3 cells, configuration 1 ; b) combined systems of 3 cells, configuration 2 ; c) systems of 4 cells.] qui correspond aux dessins (2f) à (2j) de la figure 1 reprend les combinaisons précédentes dans lesquelles nous avons effectué une permutation des matériaux; en particulier, la cellule 1 est remplacée par la cellule 3, la 2 par la 1 et la 3 par la 2 . Il faut alors noter que la courbe idéale de transmittance $T_{\mathrm{r}}$ du miroir doit avoir l'allure représentée sur la figure $2 b$.

Enfin, nous avons considéré des systèmes à quatre cellules représentés sur les dessins (2k) à (2o) de la figure 1. La courbe correspondante de transmittance $T_{\mathrm{r}}$ du miroir est représentée sur la figure $2 \mathrm{c}$. Le dessin (2k) fait intervenir 2 tandems indépendants, (21) est un double tandem couplé électriquement séparément tandis que $(2 \mathrm{~m})$ a la même configuration mais avec une liaison électrique entre les deux tandems; (2n) est une double cascade et (2o) fait intervenir une liaison électrique entre les deux cascades.

Etant donné que les matériaux comme $\mathrm{Si}, \mathrm{Ge}$, AsGa sont actuellement assez bien connus, nous avons alors développé une analyse ayant pour but de mettre en évidence les modifications apportées par l'utilisation de ces matériaux dans les systèmes précédemment décrits. Nous avons étudié des groupements de deux cellules en considérant que la photopile de plus faible bande interdite était soit AsGa, soit $\mathrm{Si}$, soit $\mathrm{Ge}$. Dans le cas de système à trois cellules, AsGa a toujours été considéré comme le matériau intermédiaire $E g_{2}$.

Dans le paragraphe 2 de cet article, nous présenterons le modèlë adopté. Dans le paragraphe 3 , nous exposerons la méthode utilisée pour le calcul des valeurs optimales de $E g_{k}$ et des maximums de rendement de nos systèmes. Dans le paragraphe 4 , nous donnerons les résultats obtenus et nous effectuerons une discussion lorsqu'il sera nécessaire.

\section{Modèle d'étude.}

Etant donné que nous étudions des systèmes de cellules ayant $E g_{1}>\cdots>E g_{k-1}>E g_{k}>\cdots>E g_{n}$ répartis régulièrement dans le spectre solaire, la première cellule absorbera donc la partie du spectre d'énergie supérieure ou égale à $E g_{1}$, la deuxième cellule la partie du spectre d'énergie comprise entre $E g_{1}$ et $E g_{2}$, la $k$-ième cellule, la partie du spectre comprise entre $E g_{k-1}$ et $E g_{k}$.

Cette absorption sélective de la lumière ainsi que le mode de couplage des cellules entraîneront différentes composantes de courant lumière que nous nous proposons d'établir; mais tout d'abord, donnons la loi du comportement de la jonction p-n.

2.1 Caractéristique courant-tension. - Nous avons supposé que le comportement photovoltaique des cellules solaires est bien décrit par l'expression suivante :

$$
J=J_{\mathrm{L}}-J_{0} \cdot\left[\exp \left(\frac{q V}{k T}\right)-1\right]
$$


avec $T=300 \mathrm{~K}$; les effets thermiques n'ont donc pas été considérés dans ce travail. $J_{0}$ et $J_{\mathrm{L}}$ dépendent étroitement des matériaux utilisés.

Pour la cellule $\mathrm{n}^{\circ} k$, la densité du courant de saturation $J_{0 k}$ est donnée par la formule suivante [1] :

$$
J_{0 k}=A_{0} \cdot \exp \left(-\frac{E g_{k}}{k T}\right) .
$$

Normalement $A_{0}$ varie suivant le type de matériau; nous l'avons maintenu constant et égal à $3 \times 10^{11} \mathrm{~A} \cdot \mathrm{m}^{-2}$ d'après [1]. Cependant, dans l'analyse des systèmes avec des matériaux réels tels que $\mathrm{Ge}, \mathrm{Si}, \mathrm{AsGa}$, nous avons développé une étude dans laquelle $A_{0}$ n'est pas constant. Les résultats sont reportés au paragraphe 4.8.

La densité de courant lumière $J_{L}$ est évidemment différente suivant les modes de couplage optique entre cellules, qui entraînent des pertes optiques différentes. Nous allons maintenant donner les expressions de $J_{\mathrm{L}}$.

2.2 EXPRESSIONS DES COURANTS DE LUMIÈRE SUIVANT LES MODES DE COUPLAGE. - Nous distinguerons quatre cas de couplage.
2.2.1 Cas idéal : aucune perte optique [1]. — La densité de courant lumière de la première cellule est donnée par :

$$
J_{\mathrm{L} 1}=q \cdot C R \cdot \int_{E g_{1}}^{\infty} N_{\mathrm{ph}}(h v) \cdot \mathrm{d} h v
$$

- La densité de courant lumière de la cellule $k$ est donnée par l'expression suivante :

$$
J_{\mathrm{L} k}=q \cdot C R \cdot \int_{E g_{k}}^{E g_{k-1}} N_{\mathrm{ph}}(h v) \cdot \mathrm{d} h v .
$$

On suppose ici un rendement de collectage unité dans les photopiles.

2.2.2 Mode tandem. - Les cellules sont placées les unes derrière les autres, celle ayant $E g_{1}$ est placée du côté des rayons incidents. Dans ce mode, chaque cellule possède un taux d'ombrage $\rho_{k}$ une réflectance superficielle $r_{k}$ et un coefficient de transmission $\tau_{k}$ qui influent sur le nombre des photons incidents; les expressions des courants lumière deviennent [4] :

- pour la première cellule :

$$
J_{\mathrm{L} 1}=q \cdot C R \cdot \int_{E g_{1}}^{\infty} N_{\mathrm{ph}}(h v) \cdot\left(1-\rho_{1}\right) \cdot\left(1-r_{1}\right) \cdot \mathrm{d} h v
$$

— pour la $k$-ième cellule :

$$
J_{\mathrm{L} k}=q \cdot C R \cdot \int_{E g_{k}}^{\infty} N_{\mathrm{ph}}(h v) \cdot\left[\prod_{i=1}^{k-1} \tau_{i}\right] \cdot\left[\prod_{i=1}^{k}\left(1-\rho_{i}\right) \cdot\left(1-r_{i}\right)\right] \cdot \mathrm{d} h v .
$$

Dans nos calculs, nous avons toujours pris :

$$
\begin{aligned}
\rho_{k} & =0,05 ; r_{k}=0,05 \\
\tau_{k} & =0,90 \text { si l'énergie du photon } h v<E g_{k} \\
\tau_{k} & =0 \quad \text { si l'énergie du photon } h v \geqslant E g_{k} .
\end{aligned}
$$

2.2.3 Mode dichroíque. - Dans ce mode, le spectre solaire est découpé par des miroirs dichroïques, chaque miroir envoyant une partie du spectre solaire sur la cellule correspondante. On doit tenir compte de la transmittance $T_{\text {rk }}$ du $k$-ième miroir.

Pour les systèmes mixtes à un miroir de la figure 1, nous avons déjà donné figure 2 les allures que doivent avoir les courbes de transmittance en fonction de l'énergie du photon.

Pour l'étude des systèmes simples de la figure 1 , nous avons pris une courbe de transmittance idéale où :

$$
\begin{aligned}
& T_{\mathrm{rk}}=1 \text { pour l'énergie du photon } h v<E g_{k} \\
& T_{\mathrm{rk}}=0 \text { si l'énergie du photon } h v \geqslant E g_{k} .
\end{aligned}
$$

Dans cette configuration, les expressions du courant lumière sont [4] :

— pour la première cellule :

$$
J_{\mathrm{L} 1}=q \cdot C R \cdot \int_{E g_{1}}^{\infty} N_{\mathrm{ph}}(h v) \cdot\left(1-T_{\mathrm{r} 1}\right) \cdot\left(1-\rho_{1}\right) \cdot\left(1-r_{1}\right) \cdot \mathrm{d} h v
$$


- pour la $k$-ième cellule :

$$
J_{\mathrm{L} k}=q \cdot C R \cdot \int_{E g_{k}}^{\infty} N_{\mathrm{ph}}(h v) \cdot\left(\prod_{i=1}^{k-1} T_{\mathrm{r} i}\right) \cdot\left(1-T_{\mathrm{rk}}\right) \cdot\left(1-\rho_{k}\right) \cdot\left(1-r_{k}\right) \cdot \mathrm{d} h v .
$$

2.2.4 Mode cascade. - Dans ce mode, on considère que les cellules sont construites en un seul bloc et peuvent être reliées par des jonctions tunnel [5] ou possèdent leur propre système de grille [6].

Nous avons considéré le premier cas en supposant que seule la première cellule a un taux d'ombrage $\rho_{1}$ et une réflectance superficielle $r_{1}$.

Les expressions des courants lumière sont :

- pour la première cellule,

$$
J_{\mathrm{L} 1}=q \cdot C R \cdot \int_{E g_{1}}^{\infty} N_{\mathrm{ph}}(h v) \cdot\left(1-\rho_{1}\right) \cdot\left(1-r_{1}\right) \cdot \mathrm{d} h v
$$

- pour la cellule $k$,

$$
J_{\mathrm{L} k}=q \cdot C R \cdot \int_{E g_{k}}^{\infty} N_{\mathrm{ph}}(h v) \cdot\left(\prod_{i=1}^{k-1} \tau_{i}\right) \cdot\left(1-\rho_{1}\right) \cdot\left(1-r_{1}\right) \cdot \mathrm{d} h v
$$

\subsection{RENDEMENTS. - Le rendement de la cellule $k$} est :

$$
\eta_{k}=\frac{V_{\mathrm{Mk}} \cdot J_{\mathrm{M} k}}{P_{\mathrm{TOT}}}
$$

où $P_{\text {TOT }}$ est la puissance totale lumineuse incidente par unité de surface. $V_{\mathrm{M} k} J_{\mathrm{Mk}}$ sont les coordonnées du point de puissance maximale [1].

Le rendement d'un système de $n$ photopiles s'écrit :

$$
\eta=\sum_{k=1}^{n} \eta_{k}
$$

Suivant les modes de couplage définis précédemment, nous aurons donc différentes valeurs de rendement.

Il est utile de préciser dès maintenant que le mode dichroïque sera toujours supérieur au mode tandem car les miroirs sont supposés parfaits. Le mode cascade n'est pas équivalent au tandem couplé électriquement. Ce dernier tient compte du taux d'ombre et de la réflectance superficielle de chaque cellule. Le mode cascade ne prend en compte ces facteurs que pour la première cellule.

On sait. donc que, d'une part, le mode cascade est supérieur au mode tandem, d'autre part le mode dichroïque est supérieur au mode tandem. Rien ne s'oppose à ce que le mode cascade soit supérieur au mode dichroïque et inversement. En particulier, les rendements obtenus dépendront pour une large part $\mathrm{du}$ choix des valeurs des paramètres technologiques $r_{k} \rho_{k}$ et $\tau_{k}$

Il faut noter que nous avons aussi calculé un rendement que nous avons appelé " rendement spectral " qui ne fait pas intervenir le fonctionnement électrique de la photopile, ni les modes de couplage. Ce rendement hypothétique constitue une valeur supérieure $\mathrm{du}$ rendement et correspond à l'énergie maximale que l'on peut récupérer dans le spectre solaire par le concept des cellules multiples. Pour cela, nous avons dit que :

- la première cellule absorbe l'énergie,

$$
E_{1}=E g_{1} \cdot \int_{E g_{1}}^{\infty} N_{\mathrm{ph}}(h v) \cdot \mathrm{d} h v
$$

- la cellule $k$, l'énergie :

$$
E_{k}=E g_{k} \cdot \int_{E g_{k}}^{E g_{k}-1} N_{\mathrm{ph}}(h v) \cdot \mathrm{d} h v
$$

L'énergie absorbée par le système de $n$ cellules sera :

$$
E=\sum_{k=1}^{n} E_{k}
$$

Le rendement spectral du système est donc :

$$
\eta=\frac{\sum_{k=1}^{n} E_{k}}{E_{\text {TOT }}}
$$

où $E_{\text {TOT }}$ est l'énergie totale incidente sur le système de cellules par unité de surface.

\section{Principes du calcul.}

Dans cette partie, nous nous proposons de déterminer pour un ensemble de $n$ cellules solaires de matériaux différents, la combinaison hypothétique des valeurs 
des $E g_{1}, E g_{2}, \ldots, E g_{n}$ qui donnera le meilleur rendement pour tous les groupements représentés dans la figure 1. Une fois cette combinaison déterminée, nous pourrons trouver les matériaux les mieux adaptés pour la réalisation de notre structure multiple.

Nous avons utilisé les mesures de M. P. Thekaekara [7] qui donnent la division du spectre solaire AM1 en 188 intervalles sur une échelle d'énergie allant de 4 à $0,3 \mathrm{eV}$. Ce grand nombre d'intervalles nous permet d'avoir une grande précision dans la détermination des $E g_{k}$ et constitue une particularité de notre étude.

La procédure de recherche des valeurs des $E g_{k}$ est la même pour les cellules indépendantes et pour les cellules avec un couplage électrique. Seule intervient une différence dans l'exécution du calcul des rendements pour ces dernières où nous avons évidemment imposé qu'un même courant électrique traverse les cellules.

Examinons d'abord la méthode utilisée pour les cellules indépendantes.

Considérons par exemple une combinaison de deux cellules ayant $E g_{1}>E g_{2}$. Les deux cellules étant positionnées sur l'échelle d'énergie, on calcule le courant lumière $J_{L k}$ pour chaque cellule $k$ qui permet de déterminer les coordonnées $V_{\mathrm{M} k}$ et $J_{\mathrm{M} k}$ du point de fonctionnement où la puissance de sortie est maximale, d'où le rendement partiel $\eta_{k}$ donné par la formule (11); le rendement de la combinaison sera égal à la somme des rendements partiels, soit :

$$
\eta=\sum_{k=1}^{2} \eta_{k}
$$

On fait évoluer la valeur de $E g_{2}$ et on détermine un rendement maximum $\eta_{\mathrm{m}}$ et la combinaison $\left(E g_{1}, E g_{2}\right)$ correspondante. On déplace $E g_{1}$ sur la valeur suivante de l'échelle d'énergie et on détermine le nouveau rendement maximum $\eta_{\mathrm{m}}$ en faisant évoluer $E g_{2}$ comme précédemment. On peut donc étudier les variations de $\eta_{\mathrm{m}}$ en fonction de $E g_{1}$ comme l'illustrent les courbes en trait plein de la figure 3 dans le cas de systèmes simples à 2, 3, 4 cellules à couplage dichroïque. Pour une certaine combinaison $E g_{1}, E g_{2}$, nous obtiendrons le maximum $\eta_{\max }$ du rendement $\eta_{\mathrm{m}}$.

Le même principe de calcul a été utilisé pour évaluer les rendements lorsque les cellules sont liées entre elles par un fil conducteur ou en cascade. Il faut alors noter qu'un même courant traverse toutes les cellules et que la tension électrique totale aux bornes du dispositif sera simplement la somme des tensions aux bornes de chaque cellule.

Après avoir déterminé le point de fonctionnement $V_{\mathrm{M} k}, J_{\mathrm{M} k}$ des cellules indépendantes, nous avons imposé un courant série $I$ égal au plus petit des courants $I_{\mathrm{M} k}$ soit $I=\inf \left(I_{\mathrm{M} k}\right)$. On obtiendra une tension d'utilisation $V_{\mathbf{M} k}$ et une puissance utile

$$
P_{k}=V_{\mathrm{M} k} \cdot \inf \left(I_{\mathrm{M} k}\right) .
$$

Le rendement global sera :

$$
\eta=\frac{\sum_{k=1}^{n} P_{k}}{P_{\text {TOT }}}
$$

On effectuera ensuite la recherche du maximum de rendement selon la méthode qui vient d'être décrite. On peut encore tracer $\eta_{\mathrm{m}}=f\left(E g_{1}\right)$ et les courbes en pointillé de la figure 3 montrent les résultats obtenus pour des systèmes simples à couplage dichroïque de 2,3 et 4 cellules.

\section{Résultats.}

Les résultats seront présentés en deux grandes parties.

Dans la première partie, nous considérerons qu'il existe des associations de matériaux hypothétiques

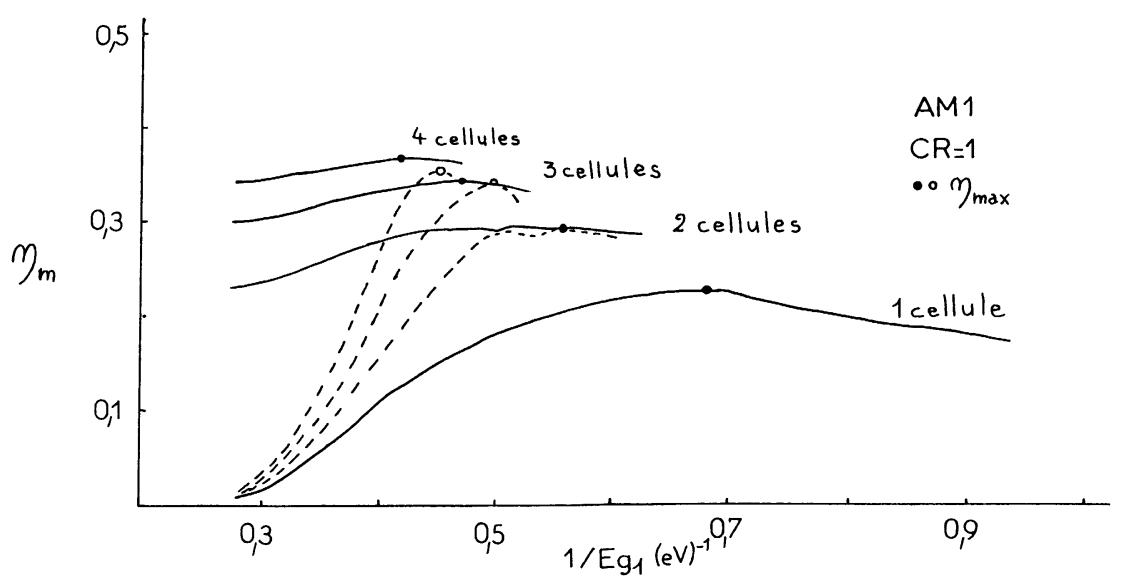

Fig. 3. - Variation du rendement $\eta_{\mathrm{m}}$ en fonction de l'inverse de la largeur de bande interdite $E g_{1}$ pour des groupements à mode dichroïque de 2, 3, 4 cellules. Traits pleins : cellules indépendantes. Traits pointillés : cellules couplées électriquement.

[Variation of the efficiency $\eta_{m}$ as a function of the inverse of the gap $E g_{1}$ for splitting systems of 2, 3, 4 cells. Solid lines : independent cells. Dashed lines : cells with electric coupling.] 
et nous donnerons les valeurs du rendement maximum $\eta_{\max }$ et des $E g_{k}$ correspondants.

Dans la deuxième partie, nous considérerons des associations avec des matériaux particuliers tels que $\mathrm{Ge}$, Si et AsGa.

$1^{\text {re }}$ partie : Etude avec des matériaux hypothétiques.

Les résultats du calcul, donnés sous forme de tableaux numérotés de I à VIII, seront présentés suivant l'ordre établi dans la figure 1, c'est-à-dire en commençant par les systèmes simples, les cellules. étant d'abord indépendantes puis ayant un couplage électrique, et en continuant par les systèmes mixtes de 3 cellules couplées par un miroir, et en terminant par les systèmes mixtes de 4 cellules couplées par un miroir.

4. 1 SYSTÈMES SIMPLES A CELLULES INDÉPENDANTES. Pour les 2 niveaux $C R$ de concentration solaire égaux à 1 et 1000 , le tableau I donne les valeurs du rendement maximum $\eta_{\max }$ et les combinaisons des $E g_{k}$ correspondánts pour des systèmes simples en mode tandem et dichroïque de 1 à 4 cellules indépendantes et représentées respectivement sur les dessins (1a) et (1b) de la figure 1 ; nous avons aussi ajouté des résultats pour les deux autres types d'association : spectrale et idéale.

L'examen du tableau permet de tirer les conclusions suivantes :

a) Si on obtient une substantielle augmentation du rendement du système en passant de 1 à 2 cellules, cette augmentation diminue au fur et à mesure que l'on ajoute des cellules. Nous nous sommes donc limités à 4 cellules bien que le principe du calcul soit valable pour un nombre quelconque de cellules.

Les systèmes à mode dichroïque sont plus performants que les systèmes à mode tandem mais les rendements que nous avons obtenus ne diffèrent pas fondamentalement des valeurs obtenues par d'autres auteurs [4].

b) Cependant, la détermination des valeurs des $E g_{k}$ est très précise du fait de la division du spectre AM1 en un nombre assez élevé d'intervalles dans

Tableau I. - Valeurs du rendement maximum $\eta_{\max }$ et des largeurs de bande interdite Eg $_{k}$; matériaux hypothétiques; systèmes simples à cellules indépendantes; (1a) et (1b) correspondent aux dessins (1a) et (1b) de la figure 1 .

[Values of the maximum efficiency $\eta_{\max }$ and gaps $E g_{k}$; hypothetical materials; simple systems with independent cells; (1a) and (1b) correspond to the pictures (1a) and (1b) of the figure 1.]

\begin{tabular}{|c|c|c|c|c|c|c|c|c|c|c|}
\hline \multirow{2}{*}{\multicolumn{2}{|c|}{ AM1 }} & \multicolumn{9}{|c|}{ Nombre de cellules } \\
\hline & & 1 & 2 & 3 & 4 & & 1 & 2 & 3 & 4 \\
\hline \multirow{4}{*}{$\begin{array}{l}\text { Mode } \\
\text { de } \\
\text { couplage }\end{array}$} & spectral & $\begin{array}{c}47,04 \\
1,127\end{array}$ & $\begin{array}{r}65,38 \\
1,486 \\
0,696\end{array}$ & $\begin{array}{c}73,37 \\
2,203 \\
1,467 \\
0,696\end{array}$ & $\begin{array}{c}79,68 \\
2,121 \\
1,486 \\
0,944 \\
0,514\end{array}$ & $\begin{array}{l}\eta_{\max }(\%) \\
E g_{1} \\
E g_{2}(\mathrm{eV}) \\
E g_{3} \\
E g_{4}\end{array}$ & $\begin{array}{c}47,04 \\
1,127\end{array}$ & $\begin{array}{c}65,38 \\
1,486 \\
0,696\end{array}$ & $\begin{array}{r}73,37 \\
2,203 \\
1,467 \\
0,696\end{array}$ & $\begin{array}{c}79,68 \\
2,121 \\
1,486 \\
0,944 \\
0,514\end{array}$ \\
\hline & idéal & $\begin{array}{l}25,06 \\
1,486\end{array}$ & $\begin{array}{c}32,83 \\
1,825 \\
1,127\end{array}$ & $\begin{array}{c}38,00 \\
2,121 \\
1,486 \\
0,963\end{array}$ & $\begin{array}{r}40,90 \\
2,433 \\
1,886 \\
1,467 \\
0,944\end{array}$ & $\begin{array}{l}\eta_{\max }(\%) \\
E g_{1} \\
E g_{2}(\mathrm{eV}) \\
E g_{3} \\
E g_{4}\end{array}$ & $\begin{array}{c}30,36 \\
1,467\end{array}$ & $\begin{array}{c}40,31 \\
1,726 \\
0,944\end{array}$ & $\begin{array}{r}46,57 \\
2,121 \\
1,486 \\
0,930\end{array}$ & $\begin{array}{c}49,49 \\
2,433 \\
1,880 \\
1,467 \\
0,930\end{array}$ \\
\hline & $\begin{array}{c}\text { tandem } \\
\text { (1a) }\end{array}$ & $\begin{array}{c}22,55 \\
1,486\end{array}$ & $\begin{array}{r}27,68 \\
1,778 \\
1,127\end{array}$ & $\begin{array}{c}29,79 \\
2,051 \\
1,467 \\
0,963\end{array}$ & $\begin{array}{c}30,51 \\
2,051 \\
1,495 \\
1,130 \\
0,929\end{array}$ & $\begin{array}{l}\eta_{\max }(\%) \\
E g_{1} \\
E g_{2}(\mathrm{eV}) \\
E g_{3} \\
E g_{4}\end{array}$ & $\begin{array}{c}27,33 \\
1,467\end{array}$ & $\begin{array}{c}34,04 \\
1,629 \\
0,944\end{array}$ & $\begin{array}{c}36,01 \\
1,909 \\
1,426 \\
0,930\end{array}$ & $\begin{array}{c}36,97 \\
2,051 \\
1,467 \\
0,973 \\
0,696\end{array}$ \\
\hline & $\begin{array}{l}\text { dichroïque } \\
\text { (1b) }\end{array}$ & $\begin{array}{c}22,55 \\
1,486\end{array}$ & $\begin{array}{c}29,35 \\
1,798 \\
1,127\end{array}$ & $\begin{array}{r}33,95 \\
2,121 \\
1,486 \\
0,963\end{array}$ & $\begin{array}{r}36,38 \\
2,460 \\
1,852 \\
1,467 \\
0,946\end{array}$ & $\begin{array}{l}\eta_{\max }(\%) \\
E g_{1} \\
E g_{2}(\mathrm{eV}) \\
E g_{3} \\
E g_{4}\end{array}$ & $\begin{array}{l}27,33 \\
1,467\end{array}$ & $\begin{array}{c}36,10 \\
1,705 \\
0,944\end{array}$ & $\begin{array}{r}41,65 \\
2,103 \\
1,467 \\
0,930\end{array}$ & $\begin{array}{c}44,07 \\
2,410 \\
1,852 \\
1,467 \\
0,930\end{array}$ \\
\hline \multicolumn{2}{|c|}{ Concentration solaire } & \multicolumn{4}{|c|}{$C R=1$} & & \multicolumn{4}{|c|}{$C R=10^{3}$} \\
\hline
\end{tabular}


l'échelle d'énergie. Il nous est ainsi possible de faire les observations suivantes :

- pour un système donné de cellules, nous avons constaté que les valeurs des $E g_{k}$ varient avec le niveau de concentration; par exemple le tableau I montre, pour deux cellules couplées en mode tandem, une variation de l'ordre de $10 \%$ pour $E g_{1}$ et de $20 \%$ pour $E g_{2}$ lorsque le niveau $C R$ de concentration varie de 1 à 1000 .

- des écarts significatifs sur les valeurs de $E g_{k}$ sont aussi enregistrés lorsqu'on passe d'un mode de couplage à un autre; par exemple le tableau I montre que pour un système de 4 cellules sous concentration $C R=10^{3}$, nous avons enregistré une variation de l'ordre de $17 \%$ pour $E g_{1}, 26 \%$ pour $E g_{2}, 50 \%$ pour $E g_{3}$ et $33 \%$ pour $E g_{4}$ lorsqu'on passe du mode tandem au mode dichroïque.

c) les $E g_{k}$ déterminés ne correspondent en général pas exactement aux valeurs rencontrées dans les matériaux réels, mais on peut sélectionner des corps dont les bandes interdites sont voisines des $E g_{k}$ calculés sans trop altérer le rendement.

Par exemple, pour des systèmes simples de 2, 3, 4 cellules indépendantes à couplage dichroïque, les courbes en trait plein de la figure 3 montrent que lorsque varie $E g_{1}$, les autres $E g_{k}$ étant toujours posi- tionnés à des valeurs donnant le rendement maximum $\eta_{\mathrm{m}}$ pour cette valeur de $E g_{1}$, le rendement $\eta_{\mathrm{m}}$ des systèmes reste assez voisin de la valeur maximale $\eta_{\max }$ de $\eta_{\mathrm{m}}$

4.2 SySTÈmes SIMPLES A COUPLAGE ÉLECTRIQUE. - Le tableau II donne les valeurs du rendement maximum $\eta_{\max }$ et des $E g_{k}$ pour, d'une part les deux systèmes simples à mode tandem et dichroïque avec couplage électrique et représentés par les dessins (1c) et (1d) de la figure 1, d'autre part les valeurs correspondant au système cascade représenté par le dessin (1e) de la figure 1. On remarque que c'est le mode dichroïque qui donne le meilleur rendement suivi par le mode cascade puis par le mode tandem. Ce dernier a d'ailleurs son rendement qui commence à décroître légèrement pour des associations de plus de 3 cellules, ceci étant dû à une absorption des photons qui croît avec le nombre de cellules.

Pour étudier l'effet du couplage électrique sur une association qui ne possédait pas de liaison électrique, effectuons une comparaison des données du tableau II par rapport à celles du tableau I. Nous remarquons que pour un même type d'association et un même nombre de cellules, le couplage électrique ne pénalise le rendement que de $7,5 \%$ au maximum pour le

Tableau II. - Valeurs du rendement maximum $\eta_{\max }$ et des largeurs de bande interdite $E g_{k}$; matériaux hypothétiques; systèmes simples de cellules avec couplage électrique; (1c), (1d), (1e) correspondent aux dessins (1c), (1d), (1e) de la figure 1.

[Values of the maximum efficiency $\eta_{\max }$ and gaps $E g_{k}$; hypothetical materials; simple systems with electric coupling; (1c), (1d), (1e) correspond to the pictures (1c), (1d), (1e) of the figure 1.]

\begin{tabular}{|c|c|c|c|c|c|c|c|c|c|c|}
\hline \multirow{2}{*}{\multicolumn{2}{|c|}{ AM1 }} & \multicolumn{9}{|c|}{ Nombre de cellules } \\
\hline & & 1 & 2 & 3 & 4 & & 1 & 2 & 3 & 4 \\
\hline \multirow{3}{*}{$\begin{array}{l}\text { Mode } \\
\text { de } \\
\text { couplage }\end{array}$} & $\begin{array}{l}\text { tandem } \\
\text { (1c) }\end{array}$ & $\begin{array}{c}22,55 \\
1,486\end{array}$ & $\begin{array}{c}27,34 \\
1,880 \\
1,147\end{array}$ & $\begin{array}{c}29,35 \\
2,121 \\
1,551 \\
0,973\end{array}$ & $\begin{array}{r}28,90 \\
2,342 \\
1,825 \\
1,379 \\
0,930\end{array}$ & $\begin{array}{l}\eta_{\max }(\%) \\
E g_{1} \\
E g_{2}(\mathrm{eV}) \\
E g_{3} \\
E g_{4}\end{array}$ & $\begin{array}{c}27,33 \\
1,467\end{array}$ & $\begin{array}{c}33,74 \\
1,748 \\
0,944\end{array}$ & $\begin{array}{r}35,68 \\
2,086 \\
1,504 \\
0,930\end{array}$ & $\begin{array}{r}34,39 \\
2,342 \\
1,825 \\
1,379 \\
0,930\end{array}$ \\
\hline & $\begin{array}{l}\text { dichroïque } \\
\text { (1d) }\end{array}$ & $\begin{array}{c}22,55 \\
1,486\end{array}$ & $\begin{array}{c}29,32 \\
1,798 \\
1,134\end{array}$ & $\begin{array}{c}33,48 \\
2,00 \\
1,486 \\
1,00\end{array}$ & $\begin{array}{c}35,34 \\
2,196 \\
1,724 \\
1,334 \\
1,00\end{array}$ & $\begin{array}{l}\eta_{\max }(\%) \\
E g_{1} \\
E g_{2}(\mathrm{eV}) \\
E g_{3} \\
E g_{4}\end{array}$ & $\begin{array}{c}27,33 \\
1,467\end{array}$ & $\begin{array}{c}36,04 \\
1,65 \\
0,944\end{array}$ & \begin{tabular}{|c}
40,82 \\
1,970 \\
1,426 \\
0,963
\end{tabular} & $\begin{array}{r}42,43 \\
2,177 \\
1,700 \\
1,273 \\
0,963\end{array}$ \\
\hline & $\begin{array}{c}\text { cascade } \\
(1 \mathrm{e})\end{array}$ & $\begin{array}{c}22,55 \\
1,486\end{array}$ & $\begin{array}{c}28,36 \\
1,825 \\
1,127\end{array}$ & $\begin{array}{r}31,59 \\
2,051 \\
1,504 \\
0,973\end{array}$ & $\begin{array}{r}32,24 \\
2,277 \\
1,778 \\
1,368 \\
0,982\end{array}$ & $\begin{array}{l}\eta_{\max }(\%) \\
E g_{1} \\
E g_{2}(\mathrm{eV}) \\
E g_{3} \\
E g_{4}\end{array}$ & $\begin{array}{c}27,33 \\
1,467\end{array}$ & $\begin{array}{c}35,03 \\
1,705 \\
0,944\end{array}$ & \begin{tabular}{|c}
38,28 \\
2,034 \\
1,467 \\
0,944
\end{tabular} & $\begin{array}{r}38,53 \\
2,277 \\
1,778 \\
1,368 \\
0,982\end{array}$ \\
\hline \multicolumn{2}{|c|}{ Concentration solaire } & \multicolumn{4}{|c|}{$C R=1$} & & \multicolumn{4}{|c|}{$C R=10^{3}$} \\
\hline
\end{tabular}


mode dichroïque; d'une manière générale, nous avons constaté tout au long de cette étude que le couplage électrique n'affecte que peu le rendement obtenu avec des cellules indépendantes.

Cependant, ce qui est important à notre avis dans les systèmes à couplage électrique, c'est la grande sensibilité du rendement $\eta_{\mathrm{m}}$ du système aux variations des valeurs des $E g_{k}$; ceci est montré dans un cas précis sur les courbes en pointillé de la figure 3 qui représentent les variations $\eta_{\mathrm{m}}=f\left(E g_{1}\right)$ de systèmes simples en mode dichroïque et à couplage électrique. Les maxima particulièrement "pointus" de ces courbes, notamment pour les combinaisons à 3 et 4 cellules, mettent en évidence clairement une diminution très rapide du rendement $\eta_{\mathrm{m}}$ lorsque $E g_{1}$ s'écarte de la valeur pour laquelle $\eta_{\mathrm{m}}=\eta_{\max }$. Nous pouvons donc dire qu'il faudra choisir des matériaux dont les valeurs des $E g_{k}$ doivent se rapprocher au mieux des valeurs calculées sous peine de voir le rendement "s'effondrer ». Heureusement, et comme le montrent les résultats du tableau II, les valeurs des $E g_{k}$ pour un même système, ne varient pas beaucoup avec le niveau $C R$ de concentration.
4. 3 Systèmes Mixtes De 3 Cellules COUPLÉES PAR UN MIROIR. - En ce qui concerne les systèmes mixtes à un miroir, les données du tableau III sont relatives à des associations de 3 cellules couplées par un miroir suivant deux types de configuration. Les différents groupements sont représentés dans la figure 1 par les dessins (2a) à (2e) pour la première configuration et par les dessins (2f) à $(2 \mathrm{j})$ pour la deuxième configuration.

On remarque qu'un ensemble de cellules assemblées suivant la configuration $\mathrm{n}^{\circ} 1$ donne toujours un meilleur rendement que le même ensemble assemblé suivant la configuration no 2 . Par exemple, pour un niveau $C R$ de concentration égal à l'unité, les 3 cellules indépendantes du dessin (2a) de la configuration $\mathrm{n}^{\mathrm{o}} 1$ conduisent à un $\eta_{\max }$ égal à $32,96 \%$, valeur légèrement supérieure à celle de $31,59 \%$ obtenue sur la structure correspondante de la configuration $\mathrm{n}^{\circ} 2$ et représentée par le dessin (2f).

On note de plus que le mode cascade permet d'obtenir le rendement le plus élevé; aussi, on trouve à $C R=1$, une valeur $\eta_{\max }=33,35 \%$ pour la configuration $\mathrm{n}^{0} 1$ où la cellule 1 est indépendante et les

Tableau III. - Valeurs du rendement maximum $\eta_{\max }$ et des largeurs de bande interdite $E g_{k}$; matériaux hypothétiques; systèmes mixtes de 3 cellules à un miroir $;(2 \mathrm{a}),(2 \mathrm{~b})$... (2j) correspondent aux dessins $(2 \mathrm{a})$... (2j) de la figure 1 .

[Values of the maximum efficiency $\eta_{\max }$ and gaps $E g_{k}$; hypothetical materials; combined systems of 3 cells and 1 mirror; (2a)... (2j) correspond to the pictures (2a)... (2j) of figure 1.]

\begin{tabular}{|c|c|c|c|c|c|c|c|c|}
\hline \multicolumn{4}{|c|}{ Configuration 1} & & \multicolumn{4}{|c|}{ Configuration 2} \\
\hline \multirow{5}{*}{$\begin{array}{l}\text { Mode de } \\
\text { couplage }\end{array}$} & $\begin{array}{l}\text { Cellules } \\
\text { indépend. } \\
\text { (2a) }\end{array}$ & $\begin{array}{c}32,96 \\
2,103 \\
1,467 \\
0,944\end{array}$ & $\begin{array}{c}40,12 \\
2,103 \\
1,467 \\
0,930\end{array}$ & $\begin{array}{l}\eta_{\max }(\%) \\
E g_{1} \\
E g_{2}(\mathrm{eV}) \\
E g_{3}\end{array}$ & $\begin{array}{c}31,59 \\
2,051 \\
1,486 \\
0,963\end{array}$ & $\begin{array}{c}38,79 \\
2,051 \\
1,481 \\
0,930\end{array}$ & $\begin{array}{l}\text { Cellules } \\
\text { indépend. } \\
\text { (2f) }\end{array}$ & \multirow{5}{*}{$\begin{array}{l}\text { Mode de } \\
\text { couplage }\end{array}$} \\
\hline & $\begin{array}{l}1 \text { indépend. } \\
2,3 \text { cascade } \\
\text { (2d) }\end{array}$ & $\begin{array}{c}33,35 \\
2,103 \\
1,495 \\
0,930\end{array}$ & $\begin{array}{c}40,82 \\
2,121 \\
1,495 \\
0,930\end{array}$ & $\begin{array}{l}\eta_{\max }(\%) \\
E g_{1} \\
E g_{2}(\mathrm{eV}) \\
E g_{3}\end{array}$ & $\begin{array}{c}32,61 \\
2,051 \\
1,495 \\
0,963\end{array}$ & $\begin{array}{c}40,01 \\
2,051 \\
1,495 \\
0,930\end{array}$ & $\begin{array}{l}3 \text { indépend. } \\
1,2 \text { cascade } \\
\text { (2i) }\end{array}$ & \\
\hline & $\begin{array}{l}1 \text { couplée } \\
\text { électriq. } \\
2,3 \text { cascade } \\
\quad \text { (2e) }\end{array}$ & $\begin{array}{r}33,11 \\
2,00 \\
1,49 \\
0,98\end{array}$ & $\begin{array}{r}40,39 \\
1,97 \\
1,44 \\
0,94\end{array}$ & $\begin{array}{l}\eta_{\max }(\%) \\
E g_{1} \\
E g_{2}(\mathrm{eV}) \\
E g_{3}\end{array}$ & $\begin{array}{r}32,36 \\
2,03 \\
1,47 \\
1,00\end{array}$ & $\begin{array}{r}39,28 \\
1,97 \\
1,34 \\
0,93\end{array}$ & $\begin{array}{l}3 \text { couplée } \\
\text { électriq. } \\
1,2 \text { cascade } \\
\quad(2 \mathrm{j})\end{array}$ & \\
\hline & $\begin{array}{l}1 \text { indépend. } \\
\quad(2 b) \\
2,3 \text { tandem } \\
\text { couplé }\end{array}$ & $\begin{array}{c}32,75 \\
2,051 \\
1,504 \\
0,930\end{array}$ & $\begin{array}{c}39,96 \\
2,068 \\
1,504 \\
0,930\end{array}$ & $\begin{array}{l}\eta_{\max }(\%) \\
E g_{1} \\
E g_{2}(\mathrm{eV}) \\
E g_{3}\end{array}$ & $\begin{array}{c}31,52 \\
2,086 \\
1,495 \\
0,963\end{array}$ & $\begin{array}{c}38,69 \\
2,086 \\
1,495 \\
0,930\end{array}$ & $\begin{array}{l}3 \text { indépend. } \\
\quad(2 \mathrm{~g}) \\
1,2 \text { tandem } \\
\text { couplé }\end{array}$ & \\
\hline & $\begin{array}{l}1 \text { couplée } \\
\text { électriq. } \\
2,3 \text { tandem } \\
\text { couplé } \\
\quad \text { (2c) }\end{array}$ & $\begin{array}{r}32,63 \\
2,00 \\
1,49 \\
0,94\end{array}$ & $\begin{array}{r}39,68 \\
2,00 \\
1,49 \\
0,94\end{array}$ & $\begin{array}{l}\eta_{\max }(\%) \\
E g_{1} \\
E g_{2}(\mathrm{eV}) \\
E g_{3}\end{array}$ & $\begin{array}{r}31,27 \\
2,07 \\
1,47 \\
1,01\end{array}$ & $\begin{array}{r}37,90 \\
2,05 \\
1,44 \\
1,00\end{array}$ & $\begin{array}{l}3 \text { couplé } \\
\text { électriq. } \\
1,2 \text { tandem } \\
\text { couplé } \\
\quad \text { (2h) }\end{array}$ & \\
\hline \multicolumn{2}{|c|}{ Concentration } & $C R=1$ & $C R=10^{3}$ & AM1 & $C R=1$ & $C R=10^{3}$ & \multicolumn{2}{|c|}{ Concentration } \\
\hline
\end{tabular}


cellules 2, 3 sont en cascade. Ce résultat est remarquable car ce rendement est tout juste inférieur à la valeur de $\eta_{\max }=33,95 \%$ donnée par un système qui paraît " plus cher » et qui est celui du couplage dichroïque de 3 cellules indépendantes par 2 miroirs; cette valeur du rendement est supérieure à la valeur $\eta_{\max }=29,79 \%$ donnée par une combinaison de 3 cellules indépendantes à couplage tandem dont le schéma est représenté par le dessin (1a) de la figure 1 pour une association de 4 cellules; elle est encore supérieure à la valeur $\eta_{\max }=31,59 \%$ donnée par un système de 3 cellules totalement en cascade et dont le schéma est représenté par le dessin (1e) de la figure 1 pour un groupement de 4 cellules; cette valeur du rendement est enfin supérieure à la valeur $\eta_{\max }=32,96 \%$ observée dans le cas où les cellules $1,2,3$ sont indépendantes et sont représentées par le dessin (2a) de la figure 1. En principe, cette association devrait donner un rendement plus grand car il n'y a aucune contrainte sur l'optimisation. Toutefois, le rendement inférieur observé provient de la réflectance et du taux d'ombrage de la cellule 3 qui pénalisent le rendement du tandem 2,3 par rapport à la cascade 2, 3 où ces grandeurs ne sont pas prises en compte.

Il est à noter que les valeurs des $E g_{k}$ des systèmes mixtes à un miroir varient peu d'une structure à une autre et ont des valeurs voisines de celles obtenues sur un système à mode dichroïque à 2 miroirs et à 3 cellules indépendantes. De plus, ces valeurs varient très peu avec le niveau de concentration par opposition aux variations beaucoup plus importantes des valeurs des $E g_{k}$ observées sur des systèmes à cellules indépendantes.

\section{4 SYSTÈMES MiXTES DE 4 CELLULES COUPLÉES PAR UN} MIROIR. - Le tableau IV donne les résultats pour des systèmes mixtes de 4 cellules couplées 2 par 2 par un miroir; on remarque que le groupement en cascade des cellules 1, 2 d'une part et 3, 4 d'autre part, représenté par le dessin (2n) de la figure 1 permet d'obtenir le rendement le plus élevé, soit une valeur de $34,56 \%$ à $C R=1$; ce rendement est bien sûr inférieur à la valeur de $36,38 \%$ donnée par une association à mode dichroïque de 3 miroirs et 4 cellules indépendantes, mais est supérieur à la valeur de $33,61 \%$ donnée par le système mixte à 1 miroir de 4 cellules indépendantes, représenté par le dessin (2k) de la figure 1. Cette dernière combinaison devrait normalement donner le meilleur rendement; toutefois, elle est pénalisée par l'utilisation des 2 montages tandems 1 , 2 et 3,4 qui font intervenir les réflectances' superficielles $r_{2}$ et $r_{4}$ ainsi que les taux d'ombrage $\rho_{2}$ et $\rho_{4}$ des cellules 2 et 4 , grandeurs qui ne sont pas prises en compte lorsqu'on utilise des montages cascade. Cependant, il faut noter que l'adjonction d'une cellule supplémentaire ne fait augmenter le rendement que faiblement par rapport aux systèmes mixtes de 3 cellules couplées par un miroir et étudiées précédemment.
Tableau IV. - Valeurs du rendement maximum $\eta_{\max }$ et des largeurs de bande interdite $E g_{k}$; matériaux hypothétiques; systèmes mixtes de 4 cellules à 1 miroir ; (2k) (2l) ... (2o) correspondent aux dessins (2k) (2l) ... (2o) de la figure 1.

[Values of the maximum efficiency $\eta_{\max }$ and gaps $E g_{k}$; hypothetical materials; combined systems of 4 cells and 1 mirror; $(2 \mathrm{k}),(21) \ldots(2 \mathrm{o})$ correspond to the pictures (2k), (2l) ... (2o) of figure 1.]

\begin{tabular}{|c|c|c|c|c|}
\hline \multirow{5}{*}{$\begin{array}{c}\text { Mode } \\
\text { de } \\
\text { couplage }\end{array}$} & $\begin{array}{l}\text { Cellules } \\
\text { indépen- } \\
\text { dantes } \\
\qquad(2 \mathrm{k})\end{array}$ & $\begin{array}{l}\eta_{\max }(\%) \\
E g_{1} \\
E g_{2}(\mathrm{eV}) \\
E g_{3} \\
E g_{4}\end{array}$ & \begin{tabular}{r|}
33,61 \\
2,277 \\
1,939 \\
1,467 \\
0,944
\end{tabular} & $\begin{array}{r}40,60 \\
2,320 \\
2,051 \\
1,467 \\
0,930\end{array}$ \\
\hline & $\begin{array}{l}\text { 1, } 2 \text { cascade } \\
\text { 3, } 4 \text { cascade } \\
\text { (2n) }\end{array}$ & $\begin{array}{l}\eta_{\max }(\%) \\
E g_{1} \\
E g_{2}(\mathrm{eV}) \\
E g_{3} \\
E g_{4}\end{array}$ & $\begin{array}{r}34,56 \\
2,341 \\
1,852 \\
1,379 \\
0,930\end{array}$ & $\begin{array}{r}41,87 \\
2,364 \\
1,880 \\
1,379 \\
0,930\end{array}$ \\
\hline & $\begin{array}{l}\text { 1, } 2 \text { tandem } \\
\text { couplé } \\
3,4 \text { tandem } \\
\text { couplé } \\
\quad(21)\end{array}$ & $\begin{array}{l}\eta_{\max }(\%) \\
E g_{1} \\
E g_{2}(\mathrm{eV}) \\
E g_{3} \\
E g_{4}\end{array}$ & $\begin{array}{r}33,16 \\
2,457 \\
1,939 \\
1,467 \\
0,930\end{array}$ & $\begin{array}{r}40,07 \\
2,533 \\
2,051 \\
1,495 \\
0,930\end{array}$ \\
\hline & $\begin{array}{l}\text { 1, } 2 \text { cascade } \\
\text { couplée élec- } \\
\text { triq. avec } \\
3,4 \text { cascade } \\
\quad(20)\end{array}$ & $\begin{array}{l}\eta_{\max }(\%) \\
E g_{1} \\
E g_{2}(\mathrm{eV}) \\
E g_{3} \\
E g_{4}\end{array}$ & $\begin{array}{r}34,29 \\
2,216 \\
1,705 \\
1,342 \\
1,004\end{array}$ & $\begin{array}{r}41,04 \\
2,216 \\
1,705 \\
1,342 \\
1,004\end{array}$ \\
\hline & $\begin{array}{l}\text { 1, } 2 \text { tandem } \\
\text { couplé en } \\
\text { liaison élec- } \\
\text { trique avec } \\
3,4 \text { tandem } \\
\text { couplé }(2 \mathrm{~m})\end{array}$ & $\begin{array}{l}\eta_{\max }(\%) \\
E g_{1} \\
E g_{2}(\mathrm{eV}) \\
E g_{3} \\
E g_{4}\end{array}$ & $\begin{array}{r}32,80 \\
2,256 \\
1,705 \\
1,356 \\
0,982\end{array}$ & $\begin{array}{r}39,20 \\
2,256 \\
1,705 \\
1,356 \\
0,982\end{array}$ \\
\hline AM1 & \multicolumn{2}{|c|}{ Concentration } & $C R=1$ & $C R=10^{3}$ \\
\hline
\end{tabular}

Nous constatons enfin que les valeurs des gaps $E g_{k}$ varient peu avec le niveau de concentration.

\section{$2^{\mathrm{e}}$ partie : Etude avec des matériaux particuliers.}

Les résultats de cette étude, donnés sous forme de tableaux numérotés 5 à 7 , seront présentés suivant l'ordre suivant :

- systèmes simples de 2 cellules,

- systèmes simples de 3 cellules,

- systèmes mixtes de 3 cellules à 1 miroir.

4.5 Systèmes Simples a deuX Cellules. - Le tableau $\mathrm{V}$ donne les valeurs du rendement maximum $\eta_{\max }$ et de $E g_{1}$ pour des systèmes simples à 2 cellules en mode tandem, dichroïque, cascade et dont le matériau de faible bande interdite $E g_{2}$ est soit $\mathrm{AsGa}$, 
Tableau V. - Valeurs du rendement maximum $\eta_{\max }$ et des largeurs de bande interdite $E g_{k}$; systèmes simples à 2 cellules; le matériau de faible bande interdite est soit $\mathrm{AsGa}$, soit $\mathrm{Si}$, soit $\mathrm{Ge} ;(1 \mathrm{a})$... (1e) correspondent aux dessins (1a) ... (1e) de la figure 1 .

[Values of the maximum efficiency $\eta_{\max }$ and gaps $E g_{k}$; simple systems of 3 cells; material of lower gap is either GaAs or Si or Ge; (1a)'... (1e) correspond to the pictures (1a) ... (1e) of figure 1.]

\begin{tabular}{|c|c|c|c|c|c|c|c|c|c|}
\hline \multicolumn{4}{|c|}{ Couples de matériaux } & \multicolumn{2}{|c|}{$\begin{array}{c}E g_{1} / \mathrm{AsGa} \\
E g_{2}=1,426 \mathrm{eV}\end{array}$} & \multicolumn{2}{|c|}{$\begin{array}{c}E g_{1} / \mathrm{Si} \\
E g_{2}=1,12 \mathrm{eV}\end{array}$} & \multicolumn{2}{|c|}{$\begin{array}{c}E g_{1} / \mathrm{Ge} \\
E g_{2}=0,666 \mathrm{eV}\end{array}$} \\
\hline \multirow{5}{*}{$\begin{array}{c}\text { Mode } \\
\text { de } \\
\text { couplage }\end{array}$} & \multirow{2}{*}{ dichroïque } & $\begin{array}{l}\text { Indépendant } \\
\text { (1b) }\end{array}$ & $\begin{array}{l}\eta_{\max }(\%) \\
E g_{1}(\mathrm{eV})\end{array}$ & $\begin{array}{l}29,05 \\
2,086\end{array}$ & $\begin{array}{l}34,08 \\
2,068\end{array}$ & $\begin{array}{c}29,34 \\
1,798\end{array}$ & $\begin{array}{c}35,86 \\
1,798\end{array}$ & $\begin{array}{c}24,22 \\
1,486\end{array}$ & $\begin{array}{c}33,11 \\
1,486\end{array}$ \\
\hline & & $\begin{array}{l}\text { couplage } \\
\text { électrique } \\
\text { (1d) }\end{array}$ & $\begin{array}{l}\eta_{\max }(\%) \\
E g_{1}(\mathrm{eV})\end{array}$ & $\begin{array}{c}28,72 \\
1,970\end{array}$ & $\begin{array}{l}33,66 \\
1,970\end{array}$ & $\begin{array}{c}29,31 \\
1,778\end{array}$ & $\begin{array}{c}35,83 \\
1,778\end{array}$ & $\begin{array}{c}23,98 \\
1,591\end{array}$ & $\begin{array}{c}32,98 \\
1,540\end{array}$ \\
\hline & \multirow{2}{*}{ tandem } & $\begin{array}{l}\text { Indépendant } \\
\text { (1a) }\end{array}$ & $\begin{array}{l}\eta_{\max }(\%) \\
E g_{1}(\mathrm{eV})\end{array}$ & $\begin{array}{c}26,72 \\
1,939\end{array}$ & $\begin{array}{c}31,27 \\
1,909\end{array}$ & $\begin{array}{c}27,68 \\
1,778\end{array}$ & $\begin{array}{c}33,63 \\
1,705\end{array}$ & $\begin{array}{c}23,88 \\
1,495\end{array}$ & $\begin{array}{l}31,97 \\
1,495\end{array}$ \\
\hline & & $\begin{array}{l}\text { couplage } \\
\text { électrique } \\
\text { (1c) }\end{array}$ & $\begin{array}{l}\eta_{\max }(\%) \\
E g_{1}(\mathrm{eV})\end{array}$ & $\begin{array}{c}26,63 \\
2,051\end{array}$ & $\begin{array}{l}31,05 \\
2,051\end{array}$ & $\begin{array}{c}27,29 \\
1,880\end{array}$ & $\begin{array}{c}33,03 \\
1,852\end{array}$ & $\begin{array}{c}23,16 \\
1,677\end{array}$ & $\begin{array}{c}31,41 \\
1,612\end{array}$ \\
\hline & cascade & (1e) & $\begin{array}{l}\eta_{\max }(\%) \\
E g_{1}(\mathrm{eV})\end{array}$ & $\begin{array}{c}27,65 \\
2,002\end{array}$ & $\begin{array}{l}32,40 \\
2,002\end{array}$ & $\begin{array}{c}28,36 \\
1,825\end{array}$ & $\begin{array}{c}34,53 \\
1,825\end{array}$ & $\begin{array}{c}23,69 \\
1,627\end{array}$ & $\begin{array}{c}32,30 \\
1,571\end{array}$ \\
\hline & \multicolumn{3}{|c|}{ Concentration AM1 } & $C R=1$ & $C R=10^{3}$ & $C R=1$ & $C R=10^{3}$ & $C R=1$ & $C R=10^{3}$ \\
\hline
\end{tabular}

soit $\mathrm{Si}$, soit $\mathrm{Ge}$. Pour les modes dichroïque et tandem, les cellules peuvent être indépendantes ou connectées électriquement par un fil conducteur.

L'étude effectuée dans la première partie avec les matériaux hypothétiques a montré que dans le cas, par exemple, d'un couplage optique à miroir de 2 cellules indépendantes, le rendement maximum $\eta_{\max }$ est de $29,35 \%$ à $C R=1$ et $36,10 \%$ à $C R=1000$. Sur le tableau V, nous voyons que le couplage par miroir d'un matériau hypothétique dont $E g_{1}=1,798 \mathrm{eV}$ avec du Si entraîne un $\eta_{\max }$ très voisin de cette valeur optimale, soit $29,34 \%$ à $C R=1$ et $35,86 \%$ à $C R=1000$. Ce résultat est meilleur que si nous avions pris AsGa comme matériau de faible bande interdite, puisque dans ce cas, le rendement $\eta_{\max }$ est un peu plus faible et égal à $29,05 \%$ à $C R=1$ et $34,08 \%$ à $C R=1000$. Avec Ge comme matériau de faible bande interdite $E g_{2}$, c'est bien moins bon, puisque dans ce cas, le rendement $\eta_{\max }$ est égal seulement à $24,22 \%$ à $C R=1$. Il faut cependant insister sur l'amélioration très nette du rendement du couplage $E g_{1} / \mathrm{Ge}$ sous concentration élevée; par exemple, à $C R=10^{3}$, on trouve $\eta_{\max }=33,11 \%$, valeur tout à fait compétitive par rapport à celle obtenue avec l'association $E g_{1} / \mathrm{AsGa}$.

Enfin, on remarque que quelle que soit la combinaison $E g_{1} / \mathrm{AsGa}, E g_{1} / \mathrm{Si}, E g_{1} / \mathrm{Ge}$, le meilleur rendement est obtenu par le mode dichroïque suivi par le mode cascade puis par le mode tandem.
4. 6 SystèMES SIMPLes À 3 Cellules. - Le tableau VI donne les valeurs du rendement maximum $\eta_{\max }$ et des $E g_{k}$ pour des systèmes simples de 3 cellules couplées en mode dichroïque, tandem et cascade; nous avons étudié deux cas :

- dans le premier cas, le matériau de bande interdite intermédiaire $E g_{2}$ est $\mathrm{AsGa}$ et nous donnons les valeurs $E g_{1}$ et $E g_{3}$.

- dans le deuxième cas, le matériau repéré par $E g_{2}$ est encore $\mathrm{AsGa}$, mais le matériau de bande interdite faible est $\mathrm{Ge}$.

Le choix de prendre AsGa pour $E g_{2}$ nous est dicté par les résultats de la première partie. Sur le tableau I qui correspond au cas des cellules indépendantes, on remarque pour des associations de 3 cellules, $E g_{2}$ varie entre $1,426 \mathrm{eV}$ qui correspond à $\mathrm{AsGa}$ et $1,486 \mathrm{eV}$ qui correspond approximativement à $\mathrm{Ga}_{0,95} \mathrm{Al}_{0,05} \mathrm{As}$.

Les résultats portés sur le tableau VI montrent que le mode dichroïque est le meilleur, suivi par le mode cascade puis par le mode tandem. Le meilleur rendement est obtenu par la combinaison $E g_{1} / \mathrm{AsGa} / E g_{3}$ de cellules indépendantes en mode dichroïque avec $E g_{1}=2,086 \mathrm{eV}$ et $E g_{3}=0,944 \mathrm{eV}, \eta_{\max }$ étant égal à $33,70 \%$ à $C R=1$, valeur très proche des $33,95 \%$ donnés par le système idéal de 3 cellules hypothétiques indépendantes couplées en mode dichroïque. La combinaison en mode dichroïque de cellules indépendantes $E g_{1} / \mathrm{AsGa} / \mathrm{Ge}$ est un peu moins bonne à $C R=1$, puisque nous trouvons un rendement de 
Tableau VI. - Valeurs du rendement maximum $\eta_{\max }$ et des largeurs de bande interdite E $g_{k}$; systèmes simples $\grave{a}$ 3 cellules; le matériau de bande interdite intermédiaire est $\mathrm{AsGa}$; (1a) ... (1e) correspondent aux dessins (1a) ... (1e) de la figure 1.

[Values of the maximum efficiency $\eta_{\max }$ and gaps $E g_{k}$; simple systems of 3 cells; material of intermediate gap is GaAs; (1a) ... (1e) correspond to the pictures (1a) ... (1e) of figure 1.]

\begin{tabular}{|c|c|c|c|c|c|c|c|}
\hline \multicolumn{3}{|c|}{ Systèmes de 3 matériaux } & \multicolumn{3}{|c|}{$\begin{array}{l}E g_{1} / \mathrm{AsGa} / E g_{3} \\
E g_{2}=1,426 \mathrm{eV}\end{array}$} & \multicolumn{2}{|c|}{$\begin{array}{l}E g_{1} / \mathrm{AsGa} / \mathrm{Ge} \\
E g_{2}=1,426 \mathrm{eV} \\
E g_{3}=0,666 \mathrm{eV}\end{array}$} \\
\hline \multirow{5}{*}{$\begin{array}{l}\text { Mode } \\
\text { de } \\
\text { couplage }\end{array}$} & \multirow{2}{*}{ dichroïque } & $\begin{array}{l}\text { Indépend. } \\
\text { (1b) }\end{array}$ & $\begin{array}{l}\eta_{\max }(\%) \\
E g_{1}(\mathrm{eV}) \\
E g_{3}\end{array}$ & $\begin{array}{c}33,70 \\
2,086 \\
0,944\end{array}$ & $\begin{array}{c}41,43 \\
2,086 \\
0,930\end{array}$ & $\begin{array}{l}30,65 \\
2,086\end{array}$ & $\begin{array}{l}39,58 \\
2,086\end{array}$ \\
\hline & & $\begin{array}{c}\text { couplage } \\
\text { électrique } \\
\text { (1d) }\end{array}$ & $\begin{array}{l}\eta_{\max }(\%) \\
E g_{1}(\mathrm{eV}) \\
E g_{3}\end{array}$ & $\begin{array}{c}33,37 \\
1,970 \\
0,963\end{array}$ & $\begin{array}{r}40,77 \\
1,970 \\
0,969\end{array}$ & $\begin{array}{l}30,08 \\
1,967\end{array}$ & $\begin{array}{l}37,47 \\
1,967\end{array}$ \\
\hline & \multirow{2}{*}{ tandem } & $\begin{array}{l}\text { Indépend. } \\
\text { (la) }\end{array}$ & $\begin{array}{l}\eta_{\max }(\%) \\
E g_{1}(\mathrm{eV}) \\
E g_{3}\end{array}$ & $\begin{array}{r}29,70 \\
1,939 \\
0,944\end{array}$ & $\begin{array}{c}36,01 \\
1,909 \\
0,930\end{array}$ & $\begin{array}{c}27,67 \\
1,939\end{array}$ & $\begin{array}{l}34,75 \\
1,909\end{array}$ \\
\hline & & $\begin{array}{l}\text { couplage } \\
\text { électrique } \\
\text { (1c) }\end{array}$ & $\begin{array}{l}\eta_{\max }(\%) \\
E g_{1}(\mathrm{eV}) \\
E g_{3}\end{array}$ & $\begin{array}{c}28,36 \\
2,051 \\
0,752\end{array}$ & $\begin{array}{c}35,12 \\
2,051 \\
0,776\end{array}$ & $\begin{array}{l}27,58 \\
2,051\end{array}$ & $\begin{array}{c}34,20 \\
2,051\end{array}$ \\
\hline & cascade & (1e) & $\begin{array}{l}\eta_{\max }(\%) \\
E g_{1}(\mathrm{eV}) \\
E g_{3}\end{array}$ & $\begin{array}{l}30,70 \\
2,034 \\
0,897\end{array}$ & $\begin{array}{c}37,95 \\
2,002 \\
0,897\end{array}$ & $\begin{array}{c}28,74 \\
2,002\end{array}$ & $\begin{array}{c}35,79 \\
2 ; 002\end{array}$ \\
\hline \multicolumn{4}{|c|}{ Concentration AM1 } & $C R=1$ & $C R=10^{3}$ & $C R=1$ & $C R=10^{3}$ \\
\hline
\end{tabular}

$30,65 \%$. Mais là encore, comme pour la combinaison $E g_{1} / \mathrm{Ge}$ du paragraphe précédent, nous notons une amélioration sensible du rendement du système sous forte concentration, soit une valeur de $39,58 \%$ assez voisine de $41,43 \%$ du système $E g_{1} / \mathrm{AsGa} / E g_{3}$.

4.7 Systèmes MIXTES À UN MIROIR DE 3 CELLULES. Le tableau VII donne les valeurs du rendement maximum $\eta_{\max }$ et des $E g_{1}, E g_{3}$ pour des systèmes mixtes à un miroir de 3 cellules; le matériau intermédiaire $E g_{2}$ est AsGa. Nous avons étudié deux types de configuration qui ont déjà été expliqués dans l'introduction de cet article.

Nous retrouvons ici les conclusions obtenues lors de l'étude avec les matériaux hypothétiques. Ainsi, pour des systèmes équivalents, la configuration $\mathrm{n}^{\circ} 1$ est supérieure à la configuration $\mathrm{n}^{\circ} 2$. Dans la configuration $\mathrm{n}^{\circ} 1$, le dispositif qui donne le meilleur rendement est obtenu lorsque l'on dispose les cellules 2 et 3 en cascade, la cellule 1 étant indépendante. Sous $C R=1$ le rendement est de $32,87 \%$, voisin des $33,35 \%$ obtenus avec le même dispositif mais constitué de matériaux hypothétiques. L'écart est encore moins élevé sous $C R=10^{3}$ puisque nous avons alors
$40,42 \%$ très proche des $40,82 \%$ donnés pour les matériaux hypothétiques. Comme nous l'avons déjà fait remarquer dans les paragraphes 4.3 et 4.4 , l'association qui devrait donner le meilleur rendement est celle où les cellules sont indépendantes et est représentée par le dessin (2a) de la figure 1. Cependant, elle est pénalisée par la présence du tandem 2-3 qui a un rendement inférieur à la cascade 2-3. Il n'est donc pas étonnant de trouver un $\eta_{\max }$ meilleur pour le dispositif de cellules avec 2 et 3 en cascade, la cellule 1 étant indépendante.

4.8 INFluence Des VARIations De $A_{0}$ SUIVANT LE TYPE DE MATÉRIAU. - Les matériaux comme $\mathrm{Si}, \mathrm{Ge}$, AsGa sont relativement bien connus et il est possible de faire une estimation plus précise de la constante $A_{0}$ de la formule (2) qui s'exprime par la relation suivante :

$$
\begin{aligned}
A_{0}=q \cdot N_{\mathrm{c}} & \cdot N_{\mathrm{v}} \times \\
\times & {\left[\frac{1}{N_{\mathrm{A}}} \cdot\left(\frac{D_{\mathrm{n}}}{\tau_{\mathrm{n}}}\right)^{1 / 2}+\frac{1}{N_{\mathrm{D}}} \cdot\left(\frac{D_{\mathrm{p}}}{\tau_{\mathrm{p}}}\right)^{1 / 2}\right] . }
\end{aligned}
$$


Tableau VII. - Valeurs du rendement maximum $\eta_{\max }$ et des largeurs de bande interdite Eg $_{k}$; systèmes mixtes de 3 cellules à 1 miroir; le matériau de bande interdite intermédiaire est $\mathrm{AsGa} ;(2 \mathrm{a})$... (2j) correspondent aux dessins (2a) ... (2j) de la figure 1.

[Values of the maximum efficiency $\eta_{\max }$ and gaps $E g_{k}$; combined systems of 3 cells and 1 mirror; material of intermediate gap is GaAs; $(2 \mathrm{a}) \ldots$... (2j) correspond to the pictures (2a) ... (2j) of figure 1.]

\begin{tabular}{|c|c|c|c|c|c|c|c|c|}
\hline & \multicolumn{7}{|c|}{$\begin{array}{l}\text { Système mixte de trois matériaux } \\
E g_{1} / \mathrm{AsGa} / E g_{3}-E g_{2}=1,426 \mathrm{eV}\end{array}$} & \\
\hline & $\begin{array}{l}\text { Mode de } \\
\text { couplage }\end{array}$ & \multicolumn{2}{|c|}{ Configuration 1} & & \multicolumn{2}{|c|}{ Configuration 2} & \multirow{2}{*}{\begin{tabular}{|l}
\multicolumn{1}{|c}{$\begin{array}{c}\text { Mode de } \\
\text { couplage }\end{array}$} \\
$\begin{array}{l}\text { Cellules } \\
\text { indépend. }\end{array}$
\end{tabular}} & \\
\hline$(2 a)$ & $\begin{array}{l}\text { Cellules } \\
\text { indépend. }\end{array}$ & $\begin{array}{c}32,79 \\
2,086 \\
0,944\end{array}$ & $\begin{array}{c}40,01 \\
2,086 \\
0,930\end{array}$ & $\begin{array}{l}\eta_{\max }(\%) \\
E g_{1}(\mathrm{eV}) \\
E g_{3}\end{array}$ & $\begin{array}{c}31,37 \\
1,939 \\
0,944\end{array}$ & $\begin{array}{c}38,62 \\
1,909 \\
0,930\end{array}$ & & $(2 f)$ \\
\hline$(2 d)$ & $\begin{array}{l}2,3 \text { cascade } \\
1 \text { indépend. }\end{array}$ & $\begin{array}{c}32,87 \\
1,939 \\
0,944\end{array}$ & $\begin{array}{c}40,42 \\
1,970 \\
0,930\end{array}$ & $\begin{array}{l}\eta_{\max }(\%) \\
E g_{1}(\mathrm{eV}) \\
E g_{3}\end{array}$ & $\begin{array}{c}32,17 \\
2,002 \\
0,944\end{array}$ & $\begin{array}{c}39,60 \\
2,002 \\
0,930\end{array}$ & $\begin{array}{l}1,2 \text { cascade } \\
3 \text { indépend. }\end{array}$ & $(2 \mathrm{i})$ \\
\hline$(2 e)$ & $\begin{array}{l}2,3 \text { cascade } \\
1 \text { couplée } \\
\text { électriq. }\end{array}$ & $\begin{array}{c}32,84 \\
1,970 \\
0,930\end{array}$ & $\begin{array}{c}40,26 \\
1,970 \\
0,930\end{array}$ & $\begin{array}{l}\eta_{\max }(\%) \\
E g_{1}(\mathrm{eV}) \\
E g_{3}\end{array}$ & $\begin{array}{l}32,08 \\
2,002 \\
0,982\end{array}$ & $\begin{array}{c}39,12 \\
2,002 \\
0,982\end{array}$ & $\begin{array}{l}1,2 \text { cascade } \\
3 \text { couplée } \\
\text { électriq. }\end{array}$ & $(2 \mathrm{j})$ \\
\hline$(2 b)$ & $\begin{array}{l}2,3 \text { tandem } \\
\text { couplé } \\
1 \text { indépend. }\end{array}$ & $\begin{array}{c}32,18 \\
1,880 \\
0,944\end{array}$ & $\begin{array}{c}39,51 \\
1,909 \\
0,930\end{array}$ & $\begin{array}{l}\eta_{\max }(\%) \\
E g_{1}(\mathrm{eV}) \\
E g_{3}\end{array}$ & $\begin{array}{c}31,31 \\
2,051 \\
0,944\end{array}$ & $\begin{array}{c}38,47 \\
2,051 \\
0,930\end{array}$ & $\begin{array}{l}1,2 \text { tandem } \\
\text { couplé } \\
3 \text { indépend. }\end{array}$ & $(2 \mathrm{~g})$ \\
\hline$(2 \mathrm{c})$ & $\begin{array}{l}2,3 \text { tandem } \\
\text { couplé } \\
1 \text { couplée } \\
\text { électriq. }\end{array}$ & $\begin{array}{c}31,20 \\
1,970 \\
0,789\end{array}$ & $\begin{array}{c}38,69 \\
1,970 \\
0,805\end{array}$ & $\begin{array}{l}\eta_{\max }(\%) \\
E g_{1}(\mathrm{eV}) \\
E g_{3}\end{array}$ & $\begin{array}{l}31,16 \\
2,051 \\
1,004\end{array}$ & $\begin{array}{c}37,79 \\
2,051 \\
1,004\end{array}$ & $\begin{array}{l}1,2 \text { tandem } \\
\text { couplé } \\
3 \text { couplée } \\
\text { électriq. }\end{array}$ & $(2 \mathrm{~h})$ \\
\hline & $\begin{array}{l}\text { Concentra- } \\
\text { tion AM1 }\end{array}$ & $C R=1$ & $C R=10^{3}$ & & $C R=1$ & $C R=10^{3}$ & $\begin{array}{l}\text { Concentra- } \\
\text { tion AM1 }\end{array}$ & \\
\hline
\end{tabular}

Le tableau VIII donne les valeurs numériques des paramètres physiques que nous avons utilisées. Dans cette partie de notre étude, nous avons simplement voulu illustrer l'influence de $A_{0}$ sur les résultats précédents. Pour cela, nous avons reporté dans le tableau VIIIb les valeurs de $\eta_{\max }$ et des $E g_{k}$ pour des systèmes simples à 2 cellules. Le matériau de faible bande interdite est soit $\mathrm{AsGa}$, soit $\mathrm{Si}$, soit $\mathrm{Ge}$. On note que par rapport aux valeurs du tableau $\mathrm{V}$ où $A_{0}$ a été maintenu constant, les rendements ont très sensiblement augmenté mais que $E g_{2}$ n'a pas beaucoup varié. Nous avons aussi ajouté la combinaison $\mathrm{AsGa} / \mathrm{Ge}$ qui nous semble intéressante surtout à forte concentration où nous avons remarqué une amélioration très sensible du rendement par rapport à sa valeur à $C R=1$.

\section{Conclusion.}

Le travail que nous venons de présenter a les particularités suivantes :
- une division précise du spectre AM1 en 188 intervalles échelonnés entre 4 et $0,3 \mathrm{eV}$,

- une étude du couplage électrique entre les cellules, effectué avec un simple fil conducteur,

- la prise en compte de la structure cascade,

- l'étude du couplage de trois cellules par un seul miroir.

Les résultats montrent que lorsque les miroirs dichroïques sont supposés parfaits :

- Les systèmes à cellules indépendantes et à plusieurs miroirs sont les meilleurs mais leur coût peut devenir prohibitif.

Il faut noter que pour ces systèmes, la répartition des $E g_{k}$ dans le spectre solaire dépend du niveau de concentration; de plus, lorsqu'on passe d'un mode de couplage à un autre, les valeurs des $E g_{k}$ peuvent varier dans d'assez grandes proportions et il faudra dans chaque cas reconsidérer les matériaux de départ.

- Lorsque le nombre de cellules est supérieur à 2 , il y a alors intérêt à utiliser des systèmes mixtes à un 
miroir. Le meilleur rendement est obtenu avec une association d'une cellule indépendante recevant la partie UV du rayonnement solaire couplée par un

Tableau VIIIa. - Valeurs numériques des paramètres physiques de la constante $A_{0}$.

[Numerical values of the physical parameters of the constant $A_{0}$.]

\begin{tabular}{|l|c|c|c|}
\hline & $\mathrm{Si}$ & $\mathrm{AsGa}$ & $\mathrm{Ge}$ \\
\hline$N_{\mathrm{c}}\left(\mathrm{cm}^{-3}\right)$ & $2,39 \times 10^{19}$ & $4,63 \times 10^{17}$ & $3,71 \times 10^{19}$ \\
\hline$N_{\mathrm{v}}\left(\mathrm{cm}^{-3}\right)$ & $8,85 \times 10^{18}$ & $8,85 \times 10^{18}$ & $4,96 \times 10^{18}$ \\
\hline$D_{\mathrm{n}}\left(\mathrm{cm}^{2} \cdot \mathrm{s}^{-1}\right)$ & 13,26 & 4,42 & 74 \\
\hline$D_{\mathrm{p}}\left(\mathrm{cm}^{2} \cdot \mathrm{s}^{-1}\right)$ & 0,78 & 36,6 & 4,27 \\
\hline$\tau_{\mathrm{n}}(\mathrm{s})$ & $2 \times 10^{-5}$ & $1 \times 10^{-9}$ & $1 \times 10^{-5}$ \\
\hline$\tau_{\mathrm{p}}(\mathrm{s})$ & $2 \times 10^{-7}$ & $7,46 \times 10^{-9}$ & $1 \times 10^{-6}$ \\
\hline$N_{\mathrm{A}}\left(\mathrm{cm}^{-3}\right)$ & $1 \times 10^{17}$ & $2 \times 10^{19}$ & $1 \times 10^{17}$ \\
\hline$N_{\mathrm{D}}\left(\mathrm{cm}^{-3}\right)$ & $1 \times 10^{19}$ & $6 \times 10^{17}$ & $5 \times 10^{18}$ \\
\hline
\end{tabular}

miroir avec une structure cascade recevant l'autre partie du rayonnement. Une particularité des systèmes mixtes à un miroir est que les valeurs des $E g_{k}$ varient très peu avec la concentration et lorsqu'on passe d'un système à un autre.

- On dispose à l'heure actuelle de matériaux comme $\mathrm{Ge}, \mathrm{Si}, \mathrm{AsGa}$ dont les largeurs de bande interdite sont relativement bien disposées dans le spectre solaire et qui sont bien adaptés à la réalisation des structures que nous avons étudiées.

Nous avons noté que Si couplé par un miroir avec un matériau dont $E g_{1}=1,798 \mathrm{eV}$ comme GaAlAs était un système " presque idéal " mais que les groupements utilisant Ge avaient leurs rendements très améliorés sous forte concentration.

- Le couplage électrique n'entraîne pas une grande perte dans le rendement; ce dernier est cependant très sensible à une variation des valeurs des $E g_{k}$ par rapport à celles calculées poür lesquelles le rendement est maximum.

Enfin, ce travail avait essentiellement pour but de faire une évaluation des $E g_{k}$ et de $\eta_{\max }$ de différents dispositifs multicolores; nous insisterons sur le fait que ces grandeurs et notamment le rendement $\eta_{\max }$ seront modulées plus ou moins fortement par les valeurs prises pour les paramètres réflectance $r$, taux d'ombrage $\rho$, transmittance $\tau$ de la cellule et transmittance du miroir $T_{\mathrm{r}}$.

Tableau VIIIb. - Valeurs du rendement maximum $\eta_{\max }$ et des largeurs de bande interdite $\mathrm{Eg}_{k}$; systèmes simples à 2 cellules; le matériau de faible bande interdite est soit $\mathrm{AsGa}$, soit $\mathrm{Si}$, soit $\mathrm{Ge}$; cas où $A_{0}$ dépend du matériau; (1a) ... (1e) correspondent aux dessins (1a) ... (1e) de la figure 1.

[Values of the maximum efficiency $\eta_{\max }$ and gaps $E g_{k}$; simple systems of 3 cells; material of lower gap is either GaAs or Si or Ge; constant $A_{0}$ is calculated for each material; (1a) ... (1e) correspond to the pictures (1a) ... (1e) of figure 1.]

\begin{tabular}{|c|c|c|c|c|c|c|c|c|c|c|c|}
\hline \multicolumn{4}{|c|}{ Couples de matériaux } & \multicolumn{2}{|c|}{$\begin{array}{l}E g_{1} / \mathrm{AsGa} \\
E g_{2}=1,426 \mathrm{eV}\end{array}$} & \multicolumn{2}{|c|}{$\begin{array}{l}E g_{1} / \mathrm{Si} \\
E g_{2}=1,12 \mathrm{eV}\end{array}$} & \multicolumn{2}{|c|}{$\begin{array}{l}E g_{1} / \mathrm{Ge} \\
E g_{2}=0,666 \mathrm{eV}\end{array}$} & \multicolumn{2}{|c|}{$\begin{array}{l}\mathrm{AsGa} / \mathrm{Ge} \\
E g_{1}=1,426 \mathrm{eV} \\
E g_{2}=0,666 \mathrm{eV}\end{array}$} \\
\hline \multirow{5}{*}{ 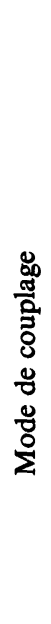 } & \multirow{2}{*}{ Dichroïque } & $\begin{array}{c}\text { Indépendant } \\
\text { (1b) }\end{array}$ & $\begin{array}{l}\eta_{\max }(\%) \\
E g_{1}(\mathrm{eV})\end{array}$ & $\begin{array}{l}33,4 \\
2,086\end{array}$ & $\begin{array}{l}38,2 \\
2,068\end{array}$ & $\begin{array}{l}33,8 \\
1,798\end{array}$ & $\begin{array}{l}40,1 \\
1,798\end{array}$ & $\begin{array}{l}35,3 \\
1,495\end{array}$ & $\begin{array}{l}39,5 \\
1,490\end{array}$ & 29,8 & 39,07 \\
\hline & & $\begin{array}{l}\text { Couplage } \\
\text { Electrique } \\
\text { (1d) }\end{array}$ & $\begin{array}{l}\eta_{\max }(\%) \\
E g_{1}(\mathrm{eV})\end{array}$ & $\begin{array}{l}33,0 \\
1,970\end{array}$ & $\begin{array}{l}38,0 \\
1,970\end{array}$ & $\begin{array}{l}33,8 \\
1,798\end{array}$ & $\begin{array}{l}40,1 \\
1,798\end{array}$ & $\begin{array}{l}35,3 \\
1,540\end{array}$ & $\begin{array}{l}39,2 \\
1,505\end{array}$ & 25,90 & 35,84 \\
\hline & \multirow{2}{*}{ Tandem } & $\begin{array}{l}\text { Indépendant } \\
\text { (1a) }\end{array}$ & $\begin{array}{l}\eta_{\max }(\%) \\
E g_{1}(\mathrm{eV})\end{array}$ & $\begin{array}{c}30,60 \\
1,909\end{array}$ & $\begin{array}{c}35,20 \\
1,778 \\
\text { à } \\
2,00\end{array}$ & $\begin{array}{c}31,92 \\
1,55 \\
\grave{a} \\
1,80\end{array}$ & $\begin{array}{c}37,93 \\
1,55 \\
\grave{a} \\
1,80\end{array}$ & $\begin{array}{c}29,63 \\
1,486\end{array}$ & $\begin{array}{c}37,95 \\
1,486\end{array}$ & 29,06 & 37,57 \\
\hline & & $\begin{array}{c}\text { Couplage } \\
\text { Electrique } \\
\text { (1c) }\end{array}$ & $\begin{array}{l}\eta_{\max }(\%) \\
E g_{1}(\mathrm{eV})\end{array}$ & $\begin{array}{c}30,43 \\
2,051\end{array}$ & $\begin{array}{c}34,86 \\
2,051\end{array}$ & $\begin{array}{c}31,14 \\
1,880\end{array}$ & $\begin{array}{l}37,0 \\
1,852\end{array}$ & $\begin{array}{c}28,86 \\
1,628\end{array}$ & $\begin{array}{c}37,06 \\
1,612\end{array}$ & 20,69 & 28,79 \\
\hline & Cascade & (1e) & $\begin{array}{l}\eta_{\max }(\%) \\
E g_{1}(\mathrm{eV})\end{array}$ & $\begin{array}{c}31,62 \\
2,002\end{array}$ & $\begin{array}{c}36,35 \\
2,002\end{array}$ & $\begin{array}{c}32,53 \\
1,825\end{array}$ & $\begin{array}{c}38,70 \\
1,825\end{array}$ & $\begin{array}{c}29,63 \\
1,591\end{array}$ & $\begin{array}{c}38,36 \\
1,551\end{array}$ & 23,32 & 32,33 \\
\hline \multicolumn{4}{|c|}{ Concentration AM1 } & $C R=1$ & $C R=10^{3}$ & $C R=1$ & $C R=10^{3}$ & $C R=1$ & $C R=10^{3}$ & $C R=1$ & $C R=10^{3}$ \\
\hline
\end{tabular}




\section{Bibliographie}

[1] LOFERSKI, J.J., Tandem photovoltaic solar cells and increased solar energy conversion efficiency, Conf. Record 12th IEEE Photovoltaic Specialists Conf., p. 957-61, Baton Rouge, La., 1976.

[2] Alvi, N.S., Backus, C.E., Masden, G.W., The potential for increasing the efficiency of photovoltaic systems by using multiple cell concepts, Conf. Record 12th IEEE Photovoltaic Specialists Conf., p. 948-56, Baton Rouge, La. 1976.

[3] Moon, R.L., James, L.W., Vander Plas, H.A., Yep, T.O., AntyPas, G.A., ChAI, Y., Multigap solar cells requirements and the performance of $\mathrm{AlGaAs}$ and $\mathrm{Si}$ cells in concentrated sunlight. Proc. 13th IEEE Photovoltaic Specialists Conf. p. 859-67, June 5-8, 1978, Washington.
[4] Bennett, A., Olsen, L.C., Analysis of multiple-cell concentrator/photovoltaic systems, Proc. 13th IEEE Photovoltaic Specialists Conf., p. 868-73, June 5-8, 1978, Washington.

[5] LAMORTE, M.F., ABBotT, D., Two-junction cascade solar cell characteristics under $10^{3}$ concentration ratio and AM0-AM 5 spectral conditions, Proc. 13th IEEE Photovoltaic Specialists Conf., p. 874-80, June 5-8, Washington.

[6] Sakai, S., Umeno, M., Theoretical analysis of new wavelenght division solar cells, J. Appl. Phys. 51 (9) (1980) 5018-24.

[7] TheKaeraKa, M.P., Solar cells book edited by Charles E. Backus (IEEE Press, New York) 1976, p. 2-5. 